\title{
The Germplasm as a Stereochemic System-I"
}

\author{
Every Individual is a Chemical Entity That Differs in Characteristic Particulars from Every Other
}

By Edward Tyson Reichert, University of Pennsylvania

THE" discovery, in 1883 , by Dr. S. Weir Mitchell and myself that the toxic principlcs of the venoms of serpents are albuminous marked an era in the chemistry, physiology and pathology of proteins, and among other thing laid the foundation of our knowledge of bacterial and other toxalbumins. Since that time our information of the properties of albuminous substances, then extremely the properties of albuminous substances, then extremely meager and somewhat chaotic, has greatly advanced, an many investigations have been made to determine the precise nature of these poisons, with the effect of more or
less modifying the statements we then set forth. Th astonishing fact that these terribly lethal substances we found by the tests of the day to be proteins, and that apart from their toxic properties they were indistinguishable frorn corresponding bodies that are ingested as food or derived therefrom by the processes of digestion, or found as normal constituents of the living tissues generally, naturally led me to much speculation and ultimately to the pursuit of the very elaborate series of remately to the pursuit of the very elaborate series of re-
searches that I have been carrying on during the past searches that $I$ have been carrying on during the past
decade under the auspices of the Carnegie Institution of Washington, reports of two of which have appeared a Publications Nos. 116 and 173.

It would be futile for me to attempt, within the necessarily restricted time that can reasonably be allotted to the reading of a communication, to present in a satisfactory form even the briefest summary of the very voluminous results and conclusions that are embodied in these works, or even an outline of their bearings, upo a vast number of problems of normal and abnormal biology, so that perforce my remarks shall be limited to a fragment-a fragment which bears upon one of the most baffling yet all-absorbing problems of life, why "like begets like."

A. THE SPHCIFICITY OF STEREOISOMERIDES

$$
\text { TO GENERA, SPECIES, ETC }
$$

These researches have as their essential basis the con ception that in different organisms corresponding comple organic substances that constitute the supreme structural components of protoplasm and the major synthetic products of protoplasmic activity are not in any case absolutely identical in chemical constitution, and that each such substance may exist in countless modifications, each modification being characteristic of the form of protoplasm, the organ, the individual, the seiv, the species and the genus. This conception was supported not only by the extraordinary differences noted between the albuminous substances venom and those of other parts of the serpent, but also by the results of the investigations of Hanriot wh described marked differences in the properties of th lipases of the pancreatic juice and the blood; of HoppeSeyler and others who stated that the pepsins of coldand warm-blooded animals are not identical; of Wr6blewsky and others who recorded differences in the pepsin of mammals; of Kossell and his students who found that the protamins obtained from the spermatozoa of different the protamins obtained from the spermatozoa of different species of fish are not identical; and of various observers
who have noted that the erythrocytes of one species when injected into the blood of another are in the nature of foreign bodies and rapidly destroyed. During subsequent years, and especially very recently, data have been rapidly accumulating along many and diverse lines of investigation which collectively indieate that every in dividual is a chemical entity that differs in characteristic divis is a chererstic the advances of biochemistry and with the trend of scientific progress toward the explanation of vital phenomen tific progress toward the explanation of vital phenomena
on a physico-chemical basis, it will be obvious that if the on a physico-chemical basis, it will be obvious that if the
conception of the non-uniform constitution of correconception of the non-uniform constitution of corre
sponding proteins and other corresponding complex or ganic substances in different organisms and parts of organisms were found to be justified by the results of laboratory investigation 'a bewildering field of speculation, reasoning and investigation would be laid open-field so' extensive as to include every domain of biological science, and seemingly to render possible, and even probable, a logical explanation of the mechanisms underlying able, a logical explanation of the mechanisms underlying
the differentiation of individuals, sex, varieties, species and genera; of the causes of fluctuations and mutation of the phenomena of Mendelism and heredity in general of the processes of fecundation and sex-determination; of the tolerance of certain organisms to organic poisons that may be extremely virulent to other forms of life; of tumor formation, reversions, malformations and monsters; of anaphylaxis, certain toxemias, immunities, etc.; and of anaphylaxis, certain toxemias, immunities, etc.; and of
vast number of other phenomena of normal and abnormal

* Read by title at the meeting of the American Philosophica and Pathological Physiology of the University of Pennsylvanis April 28th, 1914. life which as yet are partially or wholly clothed in mystery.

Some years previous to the discovery of the nature of the lethal constituents of venoms, Pastcur found that there exist three kinds of tartaric acid which, because of different effects on the ray of polarized light, are distinguished as the dextro-, lævo- and racemic-tartaric acids, the dextro form rotating the ray to the right, the lævo form to the left, and the racemic form not at all. lævo form to the left, and the racemic form not at all.
When these acids were subjected in separate solutions to When these acids were subjected in separate solutions to
the actions of Penicillium glaucum fermentation proceeded in the dextro form, but not in the lævo form, while in the solution of the racemic acid, which is a mixture of the dextro and lævo acids, the dextro form disappeared, leaving the lævo moiety unaffected. AH three acids have the same chemical composition and chemical properties but differ strikingly in their effects on polarized light and in nutritive properties. Identical or corresponding pecunumber of substances. Thus, of the twelve known forms number of substances. Thus, of the twelve known forms
of hexoses, or glucoses, only the dextro forms are fermentable, that is, capable of being used by certain low organisms as food, but not all are thus available, and, moreover, those which are show marked differences in the degree of fermentability. In the case of other substances Penicillium may consume the lævo form but not the dextro form. Other organisms show similar selectivities, using either dextro or lævo form, or both, but in the latter case in unequal degree. Evèn morè striking instances have been recorded in the actions of poisons, as for instance, dextro-nicotine is only half as toxic as the lævo form; dextro-adrenalin has only one twelfth the power of the lævo form; racemic-cocaine has a quicker and more intense but less lasting action than the løvo form; the asparagines, hyoscines, hyoscyamines and other substances have been found to exhibit marked difference in accordance with variations in their optical properties. With other bodies belonging to this category it may be found that one form is sweet while another is tasteless; another may be odorous but its enantiomorphous form without odor.

To the foregoing there may be added examples of other substances that exist in sereral forms but which physicochemically belong to a different class. Thus, nitroglycerine may exist in forms that are so different that under given conditions of temperature and percussion in substances which are found in allotropic forms may be in substances which are found in allotropic forms may be
as marked as in any of the precedirig illustrations, as for instance, in the case of phosphorus, which is familiar as the yellow, white, black and red varieties, all of which with the exception of red phosphorus are exceedingly poisonous while the latter is inert. The ortho, meta and para forms of a given substance may exhibit more or less marked physiological and toxicological variations, and so on.

The explanation of the remarkable differences shown by these substances, which differences are paralleled by those manifested by the lethal and inocuous proteins of the serpent, the pepsins, the protamins and the rediblood corpuscles, is to be found in the results of two independent but intimately related lines of physico-chemical research (1) The investigations of Van't Hoff and LeBel and'subsequent observers which have laid the foundation of a new, and to the biologist and physician, an extraordinarity important development of chemistry known as stereochemistry - a department that trèats of the arrangements of the atoms, groups and masses of molecules, or in other of the atoms, groups and masses of molecules, or in other
words of intramolecular arrangement or configuration of words of intramolecular arrangement or configuration of
molecular components in the three dimensions of space. (2) The investigations of Willard Gibbs and others which have given us the "phase rule" which defines the phases or forms in which a given substance or combination of substances may exist owing to differences in intramolecular and extramolecular arrangements and concentration of their components in relation to temperature and pressure.

According to stereochemistry a given substance may exist in multiple forms dependent upon differences in the configuration of the molecule, all of which forms have in common the fundamental chemical characteristics of a given prototype, yet each may hive certain propertie which positively distinguish it from the others. Theoretically, such substances as serum albumin, serum globu-lin, hemoglobin, starch, glycogen and chlorophyl may be produced by nature in countless modified forms owing to produced by nature in countless modified forms owing to
differences in intramolecular arrangements. Miescher differences in intramolecular arrangements. Miescher
has estimated that the serum globulin molecule may exist has estimated that the serum globulin molecule may exist
in a thousand million forms. Substances that exist in such multiple forms of a prototype are distinguished as stereisomers. The remarkable fact has been noted by Fischer and others that stereoisomers may exhibit as great or even greater differences in their properties than those manifested by even closely related isomers, which latter in comparison with stereoisomers are distantly if at all chemically related. As already instanced, so slight a change in molecular configuration as gives rise to dextro and leve forms may be sufficient to cause definite and characteristic and even profound differences in physical, nutritive and physiological properties.

In accordance with the "phase rule" a substance or a combination of substances may exist in the form of heterogeneous or homogeneous systems, a heterogeneous system consisting of a number of homogeneous systems, each of which latter is a manifestation of an individual phase and distinguishable from the others by physical, mechanical, chemical or physiological properties. The number of phases of a heterogeneous system increases with the number of component systems, and the number of the latter is in direct relationship to the number of independent variable constituents. Therefore, by means of variations of either or both intramolecular or extramolecular arrangement the number of forms of a sutstance or combination of substances may range from few to infinite.

Our means of differentiating stereoisomers are, on the whole, limited and for the most part crude, and while it has been found that differences so marked as those referred to may be detected by the ordinary procedures it seems obvious that the inherent limitations of such methods render them inadequate where a large number of stereoisomerides or related bodies which may exhibit only obscure modifications are to be definitely differentiated, so that other and more sensitive methods must be sought, or at least special methods that are adapted to exceptional conditions. The results of much preliminary investigation in this direction led in one research to the adoption of the crystallographic method, especially the use of the polarizing microscope, which in its very modern developments of analysis has demonstrated that substances which have different molecular structures exhibit corresponding differences in crystalline form and polariscopic properties; and, moreover, that the "optical reactions" may be found to be as distinctive and as exact analytically as the reactions obtained by the conventional methods of the chemist. Furthermore, the necessities of the hypothesis demanded the selection of a substance for study of a character which upon theoretical grounds might be expected to exist in nature widely distributed and readily procurable, and, as a consequence, hemoglobin was selected.

In the investigation of the hemoglobins I had as a coworker Prof. Amos Peaslee Brown. Hemoglobins were examined that were obtained from over 100 animals, representing a large variety of species, genera and families. From the data recorded certain facts are especially conspicuous, among which may be mentioned the following:

1. The comstanit recurrence of certain angles, plane and dihedral, in the hemoglobins of various species, even when the "species 'are widely separated and the crystals belong to various crystal systems. This feature indicates a common structure of the hemoglobin molecules whatever their source.

2. The constant recurrence of certain types of twinning in the hemoglobins, and the prevalence of mimosie. This has the same significance as the foregoing.

3. The constancy of generic characters in the ervstals. The crystals of the various species of any genus belong to a crystallographic group. When their characters "are tabulated they at once recall erystallographic groups'of inorganic compounds. The crystals of the genus Félis constitute an isomorphous group which is as strictly isomorphous as the groups of rhombohedral and orthiorhombic carbonates among minerals, or the more complex molecules of the members of the group of monosymmêtiric double sulphates.

4. The crystallographic specificity in relation to species. The crystals of each species of a genus, when they are favorably developed for examination in the polarizing microscope, can usually be distinguished from each othe by definite angles and other properties, while preserving the isomorphous character belonging to the genus. Where on account of difficulty of measurement the differences cannot be given a quantitative value variations in habit and mode of growth of the crystals often show specific differences.

5. The occurrence of several types of oxyhemoglobin in members of certain genera. In some species the oxyhemoglobin is dimorphous and in other trimorphous. 
Where several types of crystals occur in this way in the species of a genus the crystals of each type may be species of a genus the crystals of each type may be
arranged in an isomorphous series. In other words, cerarranged in an isomorphous series. In other words, cerand others isotrimorphous.

6. When orders, families, genera or species are wellseparated the hemoglobins are correspondingly markedly differentiated. For instance, so different are the hemoglobins of Aves, Marsupialiu, Ungulata and Rodentia that there would be no more likelihood of confounding the hemoglobins than there would be of mistaking the animals themselves. Even where there is much less zoological separation, as in the case of the genera of a given logical separation, as in the case of the genera of a given
family, but where there is well-marked zoological disfamily, but where there is well-marked zoological dis-
tinction, the hemoglobins are so different as to permit readily of positive diagnosis. When, however, the relationships are close the hemoglobins are correspondingly close, so that in instances of an alliance such as in Canis, Vulpes and Urocyon, which genera years ago were included in one genus (and doubtless correotly) the hemoglobins are very much alike, and in these cases they may globins are very much alike, and in these cases they may
exhibit closer resemblances than may be found in general exhibit closer resemblances than may be found in general
in specimens obtained from well-separated species of a genus.

So distinctive zoologically are these modified forms of hemoglobins that we had no difficulty in recognizing that the common white rat is the albino of Mus norvegicus (Mus norvegicus albus Hatai) and not of Mus rattus as almost universally stated, and that Ursidw are related to Phocidæ (as suggested by Mivart 30 years ago) but not to Canidæa as stated in modern works on zoology. Moreover, we were quick to detect errors in labeling, as for instance, when a specimen marked as coming from a
species of Papio was found to belong to one of the Felidæ. species of Papio was found to belong to one of the Felidiø.
Generic forms of hemoglobin when obtained from wellseparated genera are, in fact, so different in their molecular structures that when any two are together in solution they do not fuse to form a single kind of hemoglobin or a homogeneous solution, but continue as discrete disunited particles, so that when crystallization occurs each crystallizes independently of the other and without modification lizes independently of the other and without modification
other than that which is dependent upon such incidental other than that which is dependent upon such incidental
conditions as are to be taken into account ordinarily during erystallization. Thus, the hemoglobin of the dog crystallizes in rhombic prisms which have a diamondshaped cross-section; that of the guinea-pig in tetrahedra; that of the squirrel in hexagonal plates; and that of the rat in elongated six-sided plates. When any two of these hemoglobins are together in solution and crystallization occurs, each appears in its own form. Such phenomena indicate that the structures of the hemoglobin molecules are quite different; in fact, more differentiated than th molecules of members of an isomorphous group of simple carbonates, such as the carbonates of calcium and ma nesium which when in separate solutions crystallize in rhombohedrons whose corresponding angles differ $2 \mathrm{deg}$. 15 min., but which when in molecular union, as in the mineral dolomite, crystallize as a single substance which has an intermediate angle.

Upon the basis of our data it is not going too far to assume that it has been satisfactorily demonstrated theoretically, inferentially and experimentally that at least this one substance (hemoglobin) may exist in an inconceivable number of stereisomeric torms, ${ }^{2}$ each form being peculiar to at least genus and species and so do cidedly differentiated as to render the "hemoglabin crystal test" more sensitive in the recognition of animals and tal test" more sensitive in the recognition of animals "

animal relationships than the "zooprecipitin test."
Subsequent to the research referred to, investigation have been pursued in the study of hemoglobins from various additional sources, especially from representative of Primates, with the result in the latter case of findin indubitable evidence of an ancestral alliance of man and the man-like apes.

More or less elaborate studies by crystallographic and other methods have also been made with other albuminous substances and with starches, glycogens, phytocholesterins, chlorophyls and other complex synthetic prod lesterins, chlorophyls and other complex synthetic products of animal and plant life, especially with starches, of
whioh over 300 specimens were examined that were obwhioh over 300 specimens were examined that were ob-
tained from different plant sources, including representatained from different plant sources, including representa-
tives of a considerable number of families, genera, species, tives of a considerable number of families, genera, species results are not only in full accord with those of the hemoglobin researches but also in some instances of broader significance because by better methods of differentiation in some cases it was found possible to recognize not only in some cases it was found possible to recognize notianities as regards genus or species but also varietie peculiarities as regards genus or species but also varieties definiteness the transmission of parental characteristics.

Summing up the results of these independent but interwoven researches we find that the modified forms of each of these substances lend themselves to a very definite system of classification, and to one that is in genera accord with that of the botanist and zoologist, that is

2 Even if we assume that the different forms ary not, strictly speaking, stereoisomers it must be admitted that hemoglobin
exists in forms that are specifically modified in relation to gener and species. each genus is characterized by a distinctive type of hemoglobin, albumin, starch, etc., as the case may be, which may be designated the generic-type; every species of the genus will have a modification of this type, which is species-type, or generic primary sub-type; and ever variety of a species will have a modification of the speciestype, that is a variety-type, or generic secondary subtype, or species sub-type. In fact, it seems clear that with revisions of present classifications that are certain to come there will be found definite family types; and, moreover, that with improved methods of differentiation there will be discovered positively distinctive sex- and there will be discovered positively distinctive sex- and
individual-types. This last statement already has support in the results of collateral lines of research whic bear upon the specificities of enzymes, anaphylaxis, preipitin reactions, immune sera, etc.

From the foregoing data it seems obvious that the complex organic substances which may be assumed to constitute the essential fundamental constituents of protoplasm and the immediate complex synthetic products of proto plasmic activity may exist in exceedingly numerous or even countless stereoisomeric forms, each form being peculiarly and specifically modified in relation to genus, species, variety, race, sex, individual or even part of an individual.

PROTOPLASM A COMPLEX STEREOISOMERIC SYSTEM
The next logical step in our investigation is manifestly the study of the bearings of these stereoisomers, as such and in their variable combinations and associations, upon the structure, processes and products of protoplasm. Protoplasm according to the modern developments of biochemistry is to be regarded as being in the nature of an extremely complex, labile aggregate of proteins, fats, carbohydrates and other substances that are peculiarly associated to constitute a physico-chemical mechanism. The possible number of "phases" in which such a system can exist varies with the forms of the stereoisomerides and in general with the number and independent varia bility of the components. In such a mechanism we conceive that the number of variables is inconceivably great. From analogy we believe that such mechanisms are so extremely sensitive that the properties and processes may be modified by even so slight a change as the substitution of one form of stereoisomeride for another of the same prototype. Were it practicable to examine all of the most complex of the organic structural components of protoplasm it doubtless would be found that every one exists in a form that is peculiar to the individual and his position in classification. Moreover, we must conceiv that the components of protoplasm are as specific in relation to the form of protoplasm as are the peculia forms of stereoisomers, so that different forms of protoplasm are characterized physico-chemically (1) by the peculiarities of the stereoisomerides, and (2) by the peculiarities of the kinds, combinations, associations and arrangements

In accordance with the foregoing, the human organism may be regarded as being a highly organized composite of heterogeneous physico-chemical systems that are composed of a vast number of parts, each such part representing a particular "phase" of the system and being physically, mechanically, chemically and functionally an individual interacting unit of the aggregate. Hence, it follows that the sum or totality of these peculiarly modified stereoisomers per se, and of their arrangements with the associated components constitutes a "stereochemic system" that is peculiar to the cell; that the sum of the cell-systems is peculiar to the tissue; that the sum of the tissue-systems is peculiar to the organ; and that the sum tissue-systems is peculiar to the organ; and that the
of the organ-systems is peculiar to the individual.

of the organ-systems is peculiar to the individual.
While the living organism had been for years rec nized as being in the nature of an exceedingly complex physico-chemical aggregate of interacting independen and interdependent parts that constitute a single working unit, it has been in only recent years that the mechanism that bring about co-operative activities of the various parts has been made clear. The governing influences of the nervous system were found inadequate even in the highest organisms, not to speak of forms of life in which such actions occur but in which there is apparently a such actions occur but in which there is apparently a
total absence of nervous matter. As an associate of the nervous system, and doubtless far antedating it in or ganic evolution, is a correlative mechanism of a chemical character that is of the greatest importance, and doubtless equally so throughout the whole range of living organisms from the lowest to the highest. Every living cell whether it be in the form of a unicellular organism or a component of a multicellular organism is undoubtedly in the nature of a heterogeneous stereochemic system, each of the component parts of the system forming substances which may affect directly or indirectly the activities of the processes of the other parts; likewise every cell of a multicellular organism is not only in itself a heterogeneous system but a part of a number of associated heterogeneous systems and which by virtue of certain of its products, with or without the agency of the bloodascular or lymph-vascular systems, may exercise inluences upon other structures, which structures may
have or seemingly not have either structural or physio- logical relationship. Thus we find that a secretion formed in the pyloric glands of the gastric mucosa may excite the glands of the cardia; that growth is determined by some product or products of the pituitary body that are carried to the various structures; that the liver, pancreas and intestinal glands are excited to secretory activity by a peculiar substance formed in the duodenal and jejunal mucosæ; that carbohydrate metabolism in the liver and muscle is influenced to a profound degree by hormones that are formed in the pancreas; that lactation is determined essentially by substances derived from the corpus luteum, placenta and involuting womb; that the periods of ovulation and menstruation are inhibited by secretions of the corpus luteum; that vitally important states of activity of the generative organs are directly associated with functions of the adrenal glands; and that normal is intimately related to the ovaries and testicles. To these extraordinary correlations might be added many these extraordinary correlations might be added many
others. Some of the bodily structures are in this way so definitely associated in their activities as to constitute co-operating or interacting systems, so that the tissue products are complementary, supplementary, synergistic or antagonistic in their influences upon given structures. Such correlations must be, for perfectly obvious reasons, one of the most primitive forms of interprotoplasmic correlation, and we are justified, upun the basis of our present knowledge, in the conclusion that each active part of a cell, each cell, each tissue and each organ contributes products which may affect the activities of functionally related or unrelated parts. Hence would follow the dictum that not only is every part of a cell, every cell, every tissue and every organ an individualized stereochemic unit but also that its operations, and hence the nature of its
products, must be subject directly or indirectly to the influence of every other active part of the organism, however different the structures and functions may be.

$$
\text { ( } T \text { o be concluded.) }
$$

\section{Elevators for Eels}

A NEW method of eel-culture practised by a Dane named Nielsen is described in the annual government report on the fresh water fisheries of England and Wales. Nielsen is the proprietor of a piece of low-lying land on the Ise Fjord in the island of Zeeland, so banked in as to form a pond of about 432 acres, which is on an average about 2 feet deep. The water in the pond is somewhat brackish, and this permits of the development of abundant quantities of Mysidæ, and upon these however, that either salt water from the Fjord or fresh water from the adjacent canal can be pumped into the water from the adjacent canal can be pumped into the
lake as desired. The eel spends the greater part of its lake as desired. The eel spends the greater part of its
life in fresh water; but on attaining sexual maturity it instinctively seeks the sea, where it spawns (this statement appears to apply to all the eels of Western Europe) far out in the Atlantic in the neighborhood of the Sargasso Sea. The young eels, in their helpless become elvers, when they make their way into fresh waters, where they remain till they have reached the stage of maturity, at which nature urges them to return to the sea which gave them birth.

Nielsen observed that the elvers annually appeared in the Ise Fjord alongside his lagoon in the early spring months, and he conceived the ingenious idea of attracting them into a trap by means of a supply of fresh water, which is allowed to run through a sluice constructed in the bank of the lagoon, and thence down a sloping platform into the Fjord. He found that when he wished to discharge a volume of water through the sluice sufficient to have such an effect upon the surrounding salt water as to attract the elvers to its neighborhood, the flow was too strong to allow the fore to ascend the slope without assistance. He thereattached to the sloping platform, and allowed to drift out into the Fjord. The elvers are able to wriggle along these bands, and by means of them to make their way up the slope into a specially constructed trap, from which they are easily taken, and being counted, are transferred to the lagoon. By means of the trap, some 50,000 elvers were introduced in 1905, and in subsequent years the number has varied from about 60,000 in 1906 to about 300,000 in 1913.

When, on the other hand, Nielson requires ripe, fullygrown eels for the market, he again takes advantage of the natural instinct of the mature eel to migrate toward salt water. Salt water from the Fjord is pumped over the dam into the pond through boxes specially constructed for catching the eels, which, being

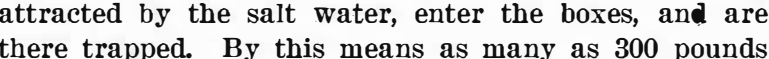
of eels have been caught in a single night, and the proprietor can take eels when he pleases. In 1913 it was estimated that the weight of eels taken was about 13,000 pounds.-The English Mechanic. 